\title{
POLARIZATION EFFECTS DUE TO COUPLING OF ELASTIC TO COMPOUND STATES
}

\author{
M.A. NAGARAJAN \\ $S E R C$, Daresbury Laboratory, Warrington WA4 $4 A D, U K$ \\ M.V. ANDRES and M. LOZANO \\ Departamento de Fisica Atómica y Nuclear, Facultad de Fisica, Universidad de Sevilla, Apartado 1065, E-41080 Sevilla, Spain
}

Received 23 January 1987; revised manuscript received 10 April 1987

\begin{abstract}
From a compound nucleus level-density-dependent imaginary potential an energy- and angular-momentum-dependent polarization potential is obtained by using the dispersion relation. The effect of coupling in this way the compound nucleus states to the elastic channel is to introduce a strongly attractive real polarization potential at small separation of the centers of mass of the colliding nuclei. The effective potential at distances around the strong absorption radii of the systems ${ }^{16} \mathrm{O}+{ }^{24} \mathrm{Mg},{ }^{28} \mathrm{Si}$, ${ }^{40} \mathrm{Ca}$ at different energies above the barrier is very slightly modified. Thus, the elastic and fusion cross sections of these reactions are hardly affected by the polarization potential.
\end{abstract}

Recently, the imaginary part of the nucleus-nucleus potential was derived under the assumption that compound nucleus formation was the only mechanism to take flux away from the elastic channel [1]. The resulting imaginary potential is strongly energy and angular momentum dependent, this dependence primarily arising from the expression for the compound nucleus level density. Treating the real part of the nucleus-nucleus potential as given by the double folding potential, the derived imaginary potential was seen to be able to reproduce the elastic scattering of several systems at energies not far above the Coulomb barrier [2].

It was shown recently that the optical potentials that describe the elastic scattering of ${ }^{16} \mathrm{O}$ by ${ }^{208} \mathrm{~Pb}$ [3] and ${ }^{60} \mathrm{Ni}$ [4] behave in an apparently anomalous fashion at energies approaching the Coulomb barrier. The imaginary potential decreases sharply in magnitude as the energy approaches the Coulomb barrier and simultaneously the real potential varies strongly with energy at these energies. This energy dependence of the real and imaginary parts of the optical potential was shown to be consistent with the

\footnotetext{
4 Work partially supported by the Spanish Comision Asesora de Investigación Científica y Técnica, contract number 2868-83.
}

dispersion relation [5] which connects the real and imaginary parts of the optical potential [6]. In general, the dispersion relation predicts that if the imaginary potential rises rapidly with energy over a small range of energy, the associated contribution to the real potential will be attractive in the same energy range.

The angular-momentum- and energy-dependent imaginary potential derived by allowing for the compound nucleus to take flux away from the elastic channel $[1,2]$ would thus be expected to give rise to a $L$-dependent and energy-dependent real polarization potential.

In this work we apply the dispersion relation to investigate this real polarization potential for the scattering of ${ }^{16} \mathrm{O}$ by ${ }^{24} \mathrm{Mg},{ }^{28} \mathrm{Si}$ and ${ }^{40} \mathrm{Ca}$.

A generalized optical potential which, incorporated in a one-body Schrödinger equation, describes the elastic scattering of two nuclei was first formally introduced for nucleon-nucleus scattering by Feshbach [5]. In general, the optical potential will be complex, non-local and energy dependent. The condition of causality (that the scattered wave cannot be emitted before the incident wave arrives) gives rise to a dispersion relation between the real and the imaginary parts of the optical potential [6]. We shall 
assume that the real and imaginary parts of the optical potential are local and write the dispersion relation as

$\Delta V(r, E)=\frac{P}{\pi} \int_{0}^{\infty} \frac{W\left(r, E^{\prime}\right)}{E^{\prime}-E} \mathrm{~d} E^{\prime}$,

where $\Delta V(r, E)$ is the polarization potential arising from the coupling of the elastic channel to other nonelastic channels.

We shall, following refs. [1,2], assume that the only channels competing with elastic scattering are the compound nucleus channels. The resulting imaginary potential can be expressed in the form

$W(r, E, L)=W(r) W(E, L)$,

where we have assumed a factorization of the radial dependence of the potential from its energy and angular-momentum dependence. The factor $W(E$, $L$ ) is assumed to be zero below the yrast line, linearly dependent on the compound nucleus level density $\rho(E, L)$ upto a constant energy above the yrast line and decreases linearly with energy to zero beyond, i.e.:

$$
\begin{gathered}
W(E, L)=0 \\
E_{\mathrm{x}}<E_{\mathrm{y}}(L), \\
=c \rho(E, L) \\
\quad E_{\mathrm{y}}(L)<E_{\mathrm{x}}<E_{\mathrm{y}}(L)+E_{1}, \\
=c_{0}+c_{1} E \\
\quad E_{\mathrm{x}}>E_{\mathrm{y}}(L)+E_{1},
\end{gathered}
$$

where $E_{\mathrm{x}}$ is the excitation energy of the compound nucleus and $E_{\mathrm{y}}(L)$ defines the yrast line. $E$ is the center of mass kinetic energy in the entrance channel. The imaginary potential, eqs. (2) and (3), is energy- and $L$-dependent. If one applies the dispersion relation that relates the real and imaginary parts of the optical potential, one would obtain an energyand $L$-dependent real polarization potential of the form

$V^{\mathrm{P}}(r, E, L)=W(r) V^{\mathrm{P}}(E, L)$,

where $V^{\mathrm{P}}(E, L)$ is defined by
$V^{\mathrm{P}}(E, L)=\frac{P}{\pi} \int_{E_{\mathrm{y}}(L)}^{c_{0} / c_{1}} \frac{W\left(E^{\prime}, L\right)}{E^{\prime}-E} \mathrm{~d} E^{\prime}$.

This polarization potential is a consequence of the coupling of the elastic channel to the compound states and represents the shift in the real potential due to these nuclear states. As it has been shown in ref. [6], small variations of the parameter $c_{1}$ hardly affect the polarization potential. The total optical potential for elastic scattering can thus be written as

$$
\begin{aligned}
& U_{\mathrm{opt}}(r, E, L)=V_{0}(r) \\
& +W(r)\left[V^{\mathrm{P}}(E, L)+\mathrm{i} W(E, L)\right],
\end{aligned}
$$

where $V_{0}(r)$ is assumed to be a double folded potential [7]. We shall investigate the properties of the polarization potential and its effects on the elastic and reaction cross section.

The systems that were studied involved the scattering of ${ }^{16} \mathrm{O}$ projectiles by ${ }^{24} \mathrm{Mg},{ }^{28} \mathrm{Si}$ and ${ }^{40} \mathrm{Ca}$ at several projectile energies. For the imaginary potential, we use the expression of the level density used in ref. [2]. The parameters for the different systems were $c_{0}=26 \mathrm{MeV}, c_{1}=-0.2$ and $E_{1}=10 \mathrm{MeV}$. The radial dependence of the imaginary part was assumed to be of a Woods-Saxon form

$W(r)=\left\{1+\exp \left[(r-R) / a_{\mathrm{I}}\right]\right\}^{-1}$,

with $R=r_{\mathrm{I}}\left(A_{1}^{1 / 3}+A_{2}^{1 / 3}\right)$. The values of $r_{\mathrm{I}}$ and $a_{1}$ are listed in table 1 as taken from ref. 2 . Using the dispersion relation, $V^{\mathrm{P}}(E, L)$ was numerically evaluated for the different projectile-target combinations. In tables 2 and 3 , we show the value of $V^{\mathrm{P}}(E, L)$ for different projectile energies and relative orbital angular momenta, $L$, for the systems ${ }^{16} \mathrm{O}+{ }^{28} \mathrm{Mg}$ and ${ }^{16} \mathrm{O}+{ }^{28} \mathrm{Si}$, respectively. The dependence of $V^{\mathrm{P}}(E, L)$ on $E$ and $L$ is shown in fig. 1 for the ${ }^{16} \mathrm{O}+{ }^{40} \mathrm{Ca}$ sys-

Table 1

The radius and diffuseness (in $\mathrm{fm}$ ) of the form factor of the imaginary potential, eq. (7), for different systems. The values are taken from ref. [2].

\begin{tabular}{lll}
\hline System & $\begin{array}{l}r_{1} \\
(\mathrm{fm})\end{array}$ & $\begin{array}{l}a_{\mathrm{I}} \\
(\mathrm{fm})\end{array}$ \\
\hline${ }^{16} \mathrm{O}+{ }^{24} \mathrm{Mg}$ & 1.274 & 0.414 \\
${ }^{16} \mathrm{O}+{ }^{28} \mathrm{Si}$ & 1.230 & 0.487 \\
${ }^{16} \mathrm{O}+{ }^{40} \mathrm{Ca}$ & 1.305 & 0.559 \\
\hline
\end{tabular}


Table 2

The energy and angular-momentum ( $L$, in units of $\hbar$ ) dependence of the real polarization potential $V^{\mathrm{P}}(E, L)$, eq. (5), for the system ${ }^{16} \mathrm{O}+{ }^{24} \mathrm{Mg}$.

\begin{tabular}{llllll}
\hline $\begin{array}{l}E_{\text {lab }} \\
(\mathrm{MeV})\end{array}$ & \multicolumn{1}{l}{$L$} & & & & \\
\cline { 2 - 6 } & 10 & 20 & 20 & 25 & 30 \\
\hline 28 & 5.84 & 11.30 & 24.44 & 10.63 & 6.58 \\
29 & 5.46 & 10.64 & 25.98 & 10.94 & 6.70 \\
30 & 5.10 & 10.02 & 27.42 & 11.27 & 6.82 \\
33 & 4.08 & 8.37 & 24.14 & 12.50 & 7.22 \\
\hline
\end{tabular}

tem. It can be noticed that for a given projectile energy, there is a characteristic value of $L$ for which the polarization potential becomes a maximum. The arrows in fig. 1 indicate the angular momentum corresponding semiclassically to the strong absorption radius, calculated as in ref. [8]. Thus, it may occur that at certain energies this angular momentum coincides with that corresponding to the maximum value of the polarization potential. In such cases, the contribution of this potential to the real part of the optical potential can be significant. The sign of the polarization potential is referred to the positive sign of $W(E, L)$ in such a way that the positive values in fig. 1 and in tables 2 and 3 mean that the dispersion relation induced an attractive correction in the real potential when the imaginary one increases. But for low partial waves $V^{\mathrm{P}}$ is repulsive. This change of character is due to the simultaneous way of energy and angular-momentum dependence of the compound nucleus level density $[1,2]$. It should be noticed that the highest energy reported in fig. 1, $139.6 \mathrm{MeV}$ for ${ }^{16} \mathrm{O}+{ }^{40} \mathrm{Ca}$, is almost in the limit of validity of the model for $W(E, L)$.

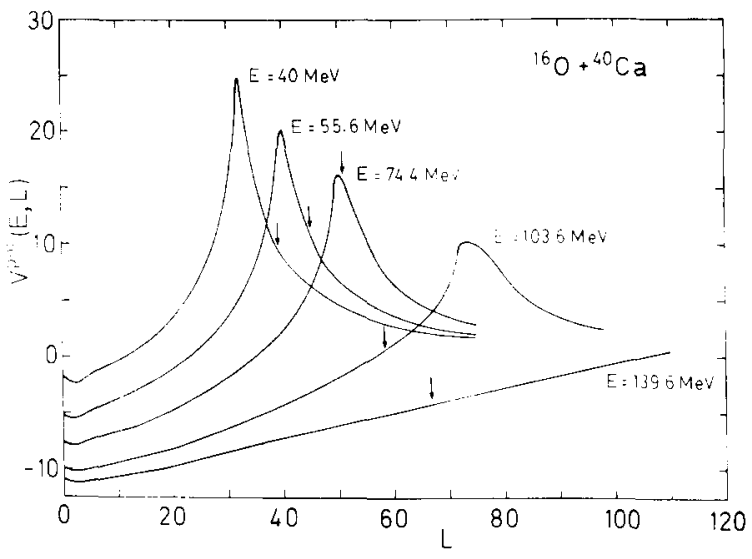

Fig. 1. The energy and angular-momentum $(L)$ dependence of the real polarization potential $V^{\mathrm{P}}(E, L)$, eq. (5), shown for different energies as a function of $L$ for the system ${ }^{16} \mathrm{O}+{ }^{40} \mathrm{Ca}$. The arrows indicate the angular momentum corresponding to the strong absorption radius calculated as in ref. [8].

In order to determine whether the effect of polarization will cause changes in the elastic scattering or the total reaction cross section, we calculated the effective real potential $V_{\text {eff }}(r, E, L)$ given by

$$
\begin{gathered}
V_{\text {eff }}(r, E, L)=V_{\text {Coul }}(r)+V_{L}(r) \\
+V_{0}(r)+W(r) V^{\mathbb{P}}(E, L),
\end{gathered}
$$

where $V_{\text {Coul }}(r)$ is the Coulomb potential and $V_{L}(r)$ is the centrifugal potential. In fig. 2 , we show the effective potential with and without the polarization potential for $33 \mathrm{MeV}{ }^{16} \mathrm{O}$ on ${ }^{28} \mathrm{Si}$ for different values of $L$. The shape of $V_{\text {eff }}$ is determined by the geometry adopted for $W(r)$. Although we have taken the parameters of table 1 because they offer the best fit to the experimental data of the elastic scattering

Table 3

The energy and angular-momentum ( $L$, in units of $\hbar)$ dependence of the real polarization potential $V^{\mathrm{P}}(E, L)$, eq. $(5)$, for the system

\begin{tabular}{|c|c|c|c|c|c|c|c|c|}
\hline \multirow{2}{*}{$\begin{array}{l}E_{\mathrm{lab}} \\
(\mathrm{MeV})\end{array}$} & \multicolumn{8}{|l|}{$L$} \\
\hline & 10 & 15 & 20 & 25 & 30 & 35 & 40 & 45 \\
\hline 33 & 5.16 & 9.48 & 28.39 & 12.19 & 7.25 & 4.92 & 3.54 & 2.65 \\
\hline 36 & 4.06 & 7.79 & 17.92 & 13.99 & 7.75 & 5.17 & 3.69 & 2.75 \\
\hline 38 & 3.38 & 6.81 & 14.87 & 15.26 & 8.13 & 5.35 & 3.79 & 2.81 \\
\hline 50 & 0.03 & 2.33 & 6.20 & 16.00 & 12.98 & 6.87 & 4.60 & 3.31 \\
\hline 66 & -3.19 & -1.59 & 0.74 & 4.20 & 11.30 & 12.67 & 6.65 & 4.40 \\
\hline 81 & -5.42 & -4.17 & -2.47 & -0.26 & 2.77 & 8.09 & 12.81 & 6.71 \\
\hline
\end{tabular}
${ }^{16} \mathrm{O}+{ }^{28} \mathrm{Si}$. 


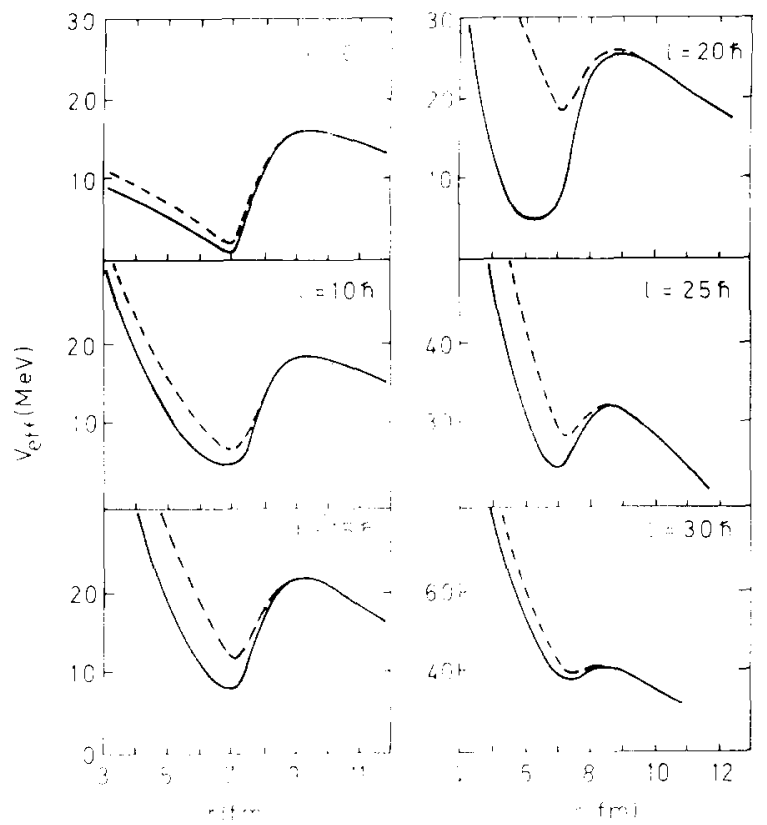

Fig. 2. The effective real potential, eq. (8), shown for different values of $L$, for $33 \mathrm{MeV}{ }^{16} \mathrm{O}$ on ${ }^{28} \mathrm{Si}$. The dotted line curve represents the effective potential and the full line curve is the effective potential with the polarization potential. The strong absorption radius calculated by the procedure of ref. [8] is 9.3 $\mathrm{fm}$; the corresponding angular momentum is $29 \hbar$.

angular distributions [1,2], we made calculations with values of $r_{1}$ and $a_{1}$ around $1.230 \mathrm{fm}$ and 0.487 $\mathrm{fm}$, respectively, for ${ }^{16} \mathrm{O}+{ }^{28} \mathrm{Si}$ at $33 \mathrm{MeV}$. The curves of fig. 2 change, as expected, for different values of the parameters but not the main features of the behavior of $V_{\text {eff. }}$ Thus, the effect of the polarization potential is significant for small $r$ (the region of large overlap of the nuclear densities) but is negligible around the strong absorption radius. This implies that the polarization potential will have no effect on the elastic scattering cross sections. This was verified by actual calculations with an optical model program modified to include the energy- and $L$-dependent polarization potential. The same result was also observed in the case of the total reaction cross sections as shown in fig. 3 . In our model, we have assumed that the loss of flux arises from a direct coupling of the elastic channel to the compound nucleus states. Hence, we could interpret the reaction cross sections as fusion cross sections. The energy dependence is of these fusion cross sections hardly affected

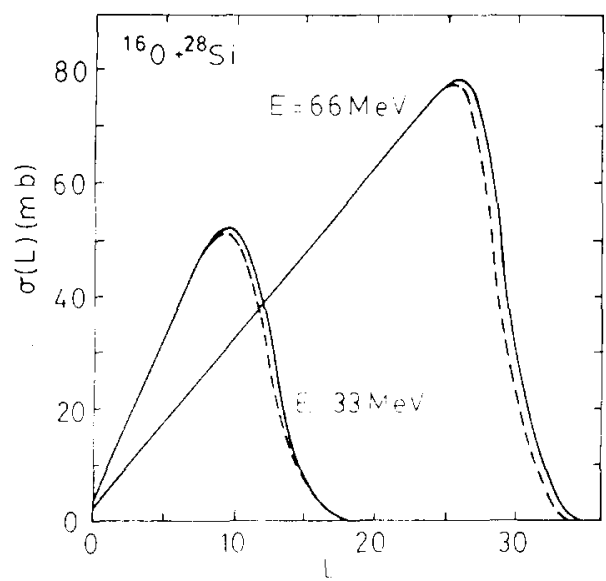

Fig. 3. The partial wave total reaction cross section for the system ${ }^{16} \mathrm{O}+{ }^{28} \mathrm{Si}$ without (dotted line) and with (full line) the real polarization potential. The corresponding energies in the laboratory system are shown on the curves.

by the polarization potential. The effect of the polarization potential on partial fusion cross sections is shown in fig. 3 for the system ${ }^{16} \mathrm{O}+{ }^{28} \mathrm{Si}$ at projectile energies of 33 and $66 \mathrm{MeV}$. Once again it is to be noticed that there is a very small effect of the polarization potential. Moreover, the models of fusion usually assume that fusion occurs after crossing the real potential barrier and hence is localized at smaller radii than implied by the $r_{\mathrm{I}}$ parametes of table 1 . The use of smaller $r_{\mathrm{I}}$ would reduce the effectiveness of the polarization potential even more.

In conclusion, we have shown that the imaginary potential due to coupling the elastic channel to the compound nuclear states provides an energydependent real polarization potential. This polarization changes its character from repulsive to attractive, depending on the bombardment energy and masses of the reacting nuclei, from low to higher partial waves. For each energy there is a particular value of the angular momentum where the polarization potential takes a maximum value. This behavior is mainly due to the way of dependence on the energy and angular momentum of the compound nucleus level density. The polarization potential hardly affects the effective potential at distances away from the strong absorption radius. Therefore the elastic and fusion cross sections are not affected by the correction introduced by the dispersion relation applied to 
a compound nucleus level-density-dependent imaginary potential.

One of the authors (M.A.N.) would like to express his thanks to Manolo Lozano for the hospitality granted to him during his visit to the University of Sevilla. Two of us (M.A.N. and M.L.) would like to thank the British Council for travel grants awarded to keep this collaboration active.

\section{References}

[1] J.M. Quesada, M. Lozano and G. Madurga, Phys. Lett. B 125 (1983) 14
[2] M.V. Andres, J.M. Quesada, M. Lozano and G. Madurga, Nucl. Phys. A 443 (1985) 380.

[3] J.S. Lilley, B.R. Fulton, M.A. Nagarajan, I.J. Thompson and D.W. Banes, Phys. Lett. B 151 (1985) 181.

[4] B.R. Fulton, D.W. Banes, J.S. Lilley, M.A. Nagarajan and I.J. Thompson, Phys. Lett. B 162 (1985) 55.

[5] H. Feshbach, Ann. Phys. (NY) 5 (1958) 357; 19 (1962) 287; R. Lipperheide, Z. Phys. 202 (1967) 88.

[6] M.A. Nagarajan, C. Mahaux and G.R. Satchler, Phys. Rev. Lett. 54 (1985) 1136;

C. Mahaux, H. Ngô and G.R. Satchler, Nucl. Phys. A 449 (1986) 354.

[7] G.R. Satchler and W.G. Love, Phys. Rep. 48 (1979) 183.

[8] G. Madurga, A. Jadraque and M. Lozano, Phys. Rev. C 23 (1981) 1536. 